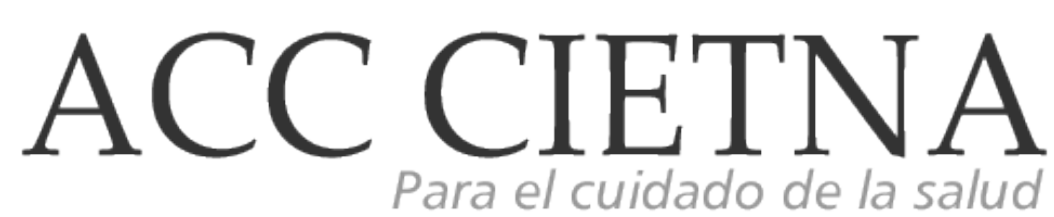

https://doi.org/10.35383/cietna.v3i2.44

\title{
Estilos de vida de las adolescentes de una Institución Educativa de Chiclayo
}

\author{
Burga Guzmán Diego Rafael ${ }^{1}$, Sandoval Rubiños Judith Eliana², Campos Bravo Yrma Lily ${ }^{3}$
}

\begin{tabular}{l} 
INFORMACIÓN DEL ARTÍCULO \\
\hline Historia del artículo: \\
Recibido el 3 de mayo de 2016 \\
Aceptado el 20 de septiembre de 2016
\end{tabular}

\section{Palabras claves:}

Adolescentes

Estilos de vida

Salud adolescente

\section{RESUMEN}

Esta investigación tuvo como objetivo identificar, analizar y comprender los estilos de vida de las adolescentes de una institución educativa de la ciudad de Chiclayo. El marco teórico estuvo basado en los conceptos de Estilos de vida y la etapa adolescente, respaldados por la Organización Mundial de la Salud (OMS), articulados a la teoría de Nola Pender. La investigación se abordó con estudio de caso, sustentado en Nisbet y Watt. Los sujetos de investigación fueron 7 adolescentes, la muestra se obtuvo por criterio de saturación; así mismo se encuestó a 74 adolescentes delimitando la muestra por conveniencia. La recolección de datos se realizó mediante entrevista abierta a profundidad, además se hizo uso del diario de campo y la encuesta como instrumentos de apoyo y fidelización de datos. El tratamiento de los datos se realizó a través del método de análisis temático según Lupicino. También se consideraron los principios éticos y de rigor científico.

Como categorías finales surgieron: "Conceptualizando sus estilos de vida", "determinando los estilos de vida en las adolescentes" y sus subcategorías. Los resultados demostraron que al ser la adolescencia una etapa conflictiva, vulnerable a los cambios por influencias, estas tienden hacia los inadecuados estilos de vida con predominio en la alimentación y sedentarismo, haciéndolas más susceptibles a tener mayores problemas de salud, siendo la familia la primera fuente de influencia en las adolescente para la obtención de estilos de vida.

\footnotetext{
1 Licenciado. Enfermero con trabajo independiente, Chiclayo, Perú. Email: burgadiego@hotmail.com

${ }^{3}$ Licenciado. Enfermera con trabajo independiente, Chiclayo, Perú.

3 Licenciada en Enfermería. Docente de la Escuela de Enfermería de la Universidad Católica Santo Toribio de Mogrovejo. Chiclayo,Perú. Email:ycampos@usat.edu.pe
} 
Lifestyles of Teenagers an educational institution - Chiclayo

\section{ABSTRACT}

Keywords:

Adolescents

Lifestyles

Health
This research entitled "The World Teen: Lifestyles of Adolescents of an Educational Institution - Chicago 2012", aimed to identify, analyze and understand their lifestyles. The theoretical framework was based on the concepts of Lifestyle and the adolescent stage, backed by the World Health Organization (WHO), articulated theory of Nola Pender. The research dealt with the case study, supported by Nisbet and Watt.

Research subjects were seven teenagers, the sample was obtained by saturation criterion, likewise surveyed 74 adolescents delimiting the convenience sample. Data collection was performed by in-depth open interviews also made use of field notes and survey as support and loyalty data. The data processing was performed using the method according Lupicinus thematic analysis. Also considered ethical principles and scientific rigor as final categories arose: "Conceptualizing their lifestyles", "determining lifestyles in adolescents" and its subcategories. The results showed that when troubled adolescence stage, vulnerable to changing influences, these tend to have a penchant for inappropriate lifestyles predominantly in food and sedentary lifestyle, making them more likely to have more health problems, being the family's primary source of influence in obtaining adolescent lifestyles.

\section{Introducción}

Concretamente, esta definición describe el estilo de vida del siguiente modo: "Forma general de vida, basada en la interacción entre las condiciones de vida en un sentido amplio y los patrones individuales de conducta determinados por factores socioculturales y características personales" 1 .

El Instituto Nacional de Estadística e Informática (INEI) reporta al 30 de junio del 2015, que en el Perú tiene 31 millones 151 mil 643 personas. Del total más de 5 millones 800 mil son adolescentes es decir, más del $20 \%$ de la población nacional. Aproximadamente el $70 \%$ viven en zonas urbanas y el $30 \%$ en zonas rurales. Más del 50 \% está en condiciones de pobreza y aproximadamente el 15 \% de los hogares de extrema pobreza están dirigidos por adolescentes y que con ello es donde empiezan los inadecuados estilos de vida sobre todo en esta etapa ${ }^{2}$.

Existe el reconocimiento de que la adolescencia al ser una etapa posterior a la niñez, es igualmente de importante, ya que alerta el hecho de que los adolescentes, sobre todo los más jóvenes, mantienen muchas actitudes y conductas de la infancia, y que el desarrollo emocional y social se produce gradualmente. Es decir, la noción de secuencia evolutiva ayuda a entender que el desarrollo previo afecta a las vulnerabilidades y recursos con los que los jóvenes afrontan los nuevos cambios del periodo adolescente. 
Es así, que la adolescencia es considerada una etapa algo más difícil que la niñez o la adultez, tanto para el adolescente como para las personas que le rodean, fundamentalmente como consecuencia de tres asuntos clave que caracterizan diferentes momentos de este periodo: conflictos con los padres; alteraciones del estado de ánimo y conductas de riesgo 3 .

Cabe mencionar que durante este periodo, los adolescentes alcanzan la madurez física y sexual, desarrollan habilidades de razonamiento más sofisticadas y toman decisiones educativas $y$ ocupacionales importantes que determinarán sus carreras adultas. Estos cambios biológicos, cognitivos y psicosociales proporcionan abundantes oportunidades de desarrollo para que los adolescentes lleven a cabo tanto conductas que pongan en riesgo su salud como conductas que revelan un estilo de vida saludable, por esto se formuló la siguiente pregunta de investigación ¿Cómo son los estilos de vida de las adolescentes de una institución Educativa de la ciudad de Chiclayo?, y cómo objetivos de investigación, identificar, analizar y comprender los estilos de vida de las adolescentes.

\section{Metodología}

Esta investigación es cualitativa4 pues se requirió de un profundo entendimiento de los estilos de vida de las adolescentes. Se trabajó con las adolescentes tanto grupal como individualmente, teniendo en cuenta sus estilos de vida, permitiéndonos describir detalladamente las situaciones, comportamientos, eventos, interacciones, actitudes, creencias y pensamientos tal y como son sentidas y expresadas por los sujetos de estudio.

El diseño empleado es el estudio de caso $^{5}$ que utiliza el aporte de técnicas de evaluación y su análisis para reflexionar y debatir en torno a las características de la muestra estudiada, considerándolas como holísticamente, cuyos atributos podemos entender en su totalidad solamente en el momento en que examinemos todos simultáneamente.

Con el previo consentimiento informado y firmado, se procedió a realizar la entrevista abierta a profundidad a las adolescentes en el mismo escenario, de acuerdo a su disponibilidad horaria, en un ambiente tranquilo (aula previamente coordinada), con una duración promedio de 20 minutos, utilizando un gravador de voz, al mismo tiempo se realizó registros en el diario de campo. Los datos se analizaron, según el análisis de contenido temático, y en todo momento se respetaron los principios de la ética personalista y los de rigor científico, de credibilidad, confirmabilidad, y transferibilidad.

\section{Resultados, análisis y discusión}

1. CONCEPTUALIZANDO SUS ESTILOS DE VIDA.

2. DETERMINADO LOS ESTILOS DE VIDA EN LAS ADOLESCENTES.

2.1.- Estilos de alimentarse.

2.2.- Estilos de descanso y sueño.

2.3.- Estilos de actividad física y deporte.

2.4.- Estilos de divertirse.

2.5.- Estilos de disponer el tiempo libre.

\section{Conceptualizando sus estilos de vida}

Estilo de vida o forma de vida designa, de una manera genérica, a la forma o manera en que se entiende la vida; tanto en el sentido de una particular concepción del mundo, una idiosincrasia o un carácter, particular o de grupo, expresado en todos o en cualquiera de los ámbitos del comportamiento (trabajo, ocio, sexo, alimentación, indumentaria, etc.), fundamentalmente en las costumbres o la vida cotidiana, como en la vivienda y el urbanismo, en la relación con los objetos y la posesión de bienes, en la relación con el entorno o en las relaciones interpersonales6. En el estudio realizado las adolescentes aportan diferentes conceptualizaciones según su opinión y 
conocimientos, que lo relacionan con sus prácticas, como se evidencia en las siguientes expresiones:

"Yo defino estilo de vida, a que tenemos que tener una vida ordenada con nuestros propios horarios para cada día, respecto a eso, siento que estoy más o menos, ya que yo no cuento aun con un horario, lo cual pienso cambiar prontamente porque es para mí beneficio..."(Clavel).

“...Para mi estilos de vida son las actividades que realizamos en nuestro diario vivir..." (Girasol).

"Estilos de vida está relacionado con el comportamiento..." (Margarita)

Como se evidencia, las adolescentes conceptualizan estilos de vida como un conjunto de actitudes, así como refiere la National Health Service, que define a los estilos de vida, como el conjunto de actitudes, conocimientos $y$ comportamientos que determinan nuestra manera de vivir ${ }^{7}$ en tal sentido en el presente estudio, algunas adolescentes relacionan a los estilos de vida, a la manera de alimentarse, a la actividad física, la diversión y con el seguimiento de algunas reglas, por influencia o recomendación médica y/o familiar, utilizando para la conceptualización de estilos de vida frases como: "es tener una vida ordenada" "es lo que realizo todos los días" "es la forma de vida, sea buena o mala" "son las actividades que realizamos para vivir" y en otros discursos refieren que está "relacionado con el comportamiento; así mismo, se evidencia que las adolescentes no tienen el conocimiento para definir estilos de vida, si no que se centran más en las necesidades que tienen que satisfacer como: la alimentación que ocupa el primer lugar de importancia, así mismo algunas de ellas presentan actitudes positivas para tratar de modificar estos estilos y un comportamiento inadecuado frente a la incorrecta toma de decisión sobres sus propios estilos de vida.

En el estudio realizado, también se develó que algunas adolescentes practican estilos de vida no saludables, pues como dice Elliot, las adolescentes no toman importancia a aquellos estilos que promueven o protegen la salud, sino a los estilos que lo ponen en riesgo, debido al deficiente conocimiento, a las actitudes negativas frente a la toma de decisiones $y$ al comportamiento incorrecto, impulsado por factores económicos, sociales $\mathrm{y} / \mathrm{o}$ familiares.

\section{Determinando los estilos de vida de las adolescentes.}

Al respecto las adolescentes, sujeto del presente estudio, lo relacionan con la satisfacción de las necesidades básicas, las mismas que corresponden a la pirámide de Maslow, quien lo clasifica en 2 tipos: necesidades de supervivencia, relacionadas con la necesidad de alimentación, descanso y sueño, etc. y las de crecimiento, relacionado con la diversión, tiempo libre, actividad y ejercicio8. Por otro lado, Virginia Henderson prioriza 14 necesidades clasificándolas de la siguiente manera: necesidades básicas en comer y beber, movimiento y postura, descanso y sueño, participación en la recreación, etc., y según María Ramos en su tesis doctoral denominada "Estilos de vida y salud en la adolescencia" quien clasifica en primer lugar a los hábitos alimenticios, actividad física, diversión/hábitos nocivos, etc.

\subsection{Estilos de alimentarse}

Durante el periodo de la adolescencia, el crecimiento es acelerado con un aumento importante de la talla entre $0.21 \mathrm{~cm}$ a $0.24 \mathrm{~cm}$ por mes, así como de masa corporal dentro de 20 a $24.9 \mathrm{Kg} / \mathrm{m}^{2}$; Es por ello, que la alimentación tiene una especial importancia debido a los altos requerimientos nutricionales que necesitan para hacer frente a los grandes cambios, pues una adolescente tiene como requerimiento nutricional entre 2400 a $2600 \mathrm{Kcal}$ en mujeres y de 2600 $2900 \mathrm{Kcal}$ en hombres, divididos en los siguientes macronutrientes $15 \%$ de proteínas, 35\% de lípidos y $55 \%$ de carbohidratos 8 . Las adolescentes, gracias a la independencia adquirida y a su capacidad 
cognitiva formal comienzan a decidir sobre varios aspectos de su vida, y la alimentación se vuelve un factor decisivo en esta etapa, porque aumenta su apetito; comer saludablemente durante la adolescencia es importante porque los grandes cambios del cuerpo durante este período afectan a las necesidades de nutrición y dieta del futuro adulto. La adecuada alimentación en esta etapa, ayudará a prevenir que el adulto sufra enfermedades como hipercolesterolemia, hipertensión arterial, obesidad y osteoporosis ${ }^{9}$.

Al respecto, Crovetto, citado por Campos, señala, que el incremento en el nivel económico de un sector de la población trajo como consecuencia un mayor acceso a un nuevo mercado alimenticio, 8 es decir, el basado en la alimentación moderna, el cual ha llevado a que el $11 \%$ de las adolescentes, en las edades de 15 a 17 años, se encuentran en riesgo de padecer algunos trastornos metabólicos. Además, se puede observar un incremento en la incidencia de infarto cardíaco y de litiasis vesicular en edades cada vez más tempranas, es decir ya no afecta solamente a personas adultas (+45 años), sino a jóvenes de 15 años a más. ${ }^{9}$

La alimentación equilibrada en las adolescentes consta de 5 comidas al día: empezando por el desayuno, que es el primer alimento importante al empezar el día; el refrigerio o entre comida, que se consume a media mañana para mantener las energías y para seguir con las actividades; el almuerzo al medio día, que forma parte importante en donde se consumen el mayor aporte calórico; el lonche o la merienda, es un tipo de comida ligera que se toma a media tarde y la cena, que es la última comida del día que se toma por la noche.

Como el primer alimento del día, está el desayuno, en el que se insiste que es una de las comidas importantes del día, porque luego de un ayuno prolongado de 8 o 10 horas de sueño es necesario recobrar energía ${ }^{10}$, sin embargo, la mayoría de las adolescentes lo omiten o no lo ingieren en el horario adecuado, tal como se evidencia en la siguiente unidad de análisis:
“...De lunes a viernes me levanto a las 6 am, me aseo, voy a comprar pan, preparo el desayuno, le sirvo a mi sobrino y también tomo yo, lavo los platos y vengo al colegio, pero a veces me levanto tarde y no desayuno, porque si lo hago llegaría tarde al colegio..." (Violeta).

“...No tomo desayuno porque sufro de gastritis y cuando como un pan me empieza a doler el estómago...Sólo tomo un vaso de leche o avena $y$ me voy al colegio..." (Gardenia).

Las adolescentes manifiestan que a veces no toman desayuno por la premura del tiempo y que según refiere Angeles, que el desayuno podría considerarse como la comida sólida consumida antes de iniciar la jornada escolar o la realizada antes de las 11:00 hrs, durante los fines de semana. También podría definirse simplemente como la primera comida del día; sin embargo, ninguna de las dos definiciones dejan entrever la importancia que tiene dentro de la dieta total.

Junto con la comida y la cena, el desayuno es también una buena fuente de energía y nutrientes; de hecho, está comprobado que sin el desayuno la alimentación difícilmente puede ser nutricionalmente correcta ${ }^{1}$.

El problema del desayuno es que las adolescentes lo omiten, siendo muchas veces reemplazados por un refrigerio consumido en la escuela, y esto se evidencia en la siguiente unidad de análisis:

“...Pero cuando no tomo desayuno llevo en mi lonchera, frutas: naranja, mandarina o plátano y a veces Sporade, no consumo en el kiosco..." (Margarita).

“...cuando no tomo desayuno, en el recreo como un plátano o manzana y a veces un chocolate porque me gustan mucho, pero no los como frecuentemente..." (Girasol).

El refrigerio o entre comidas que satisfacen $y$ llenan deben incluir los 3 macronutrientes, carbohidratos, proteínas y grasas. Los 
carbohidratos proveen de volumen y valor energético, las proteínas y las grasas dan saciedad, por eso según Mataix Verdu el refrigerio debe aportar el $10 \%$ del total de los requerimientos nutricionales (2400 a $2600 \mathrm{Kcal}$ ), es decir un equivalente a $250 \mathrm{Kcal}{ }^{10}$.

En tal sentido, podemos verificar que en las unidades de análisis anteriores existe un exceso de la real aportación de Kcal sobre todo en azúcares, pues la mayoría de adolescentes manifestaron que consumen gelatina, que contiene $60 \mathrm{Kcal}$, chocolates $290 \mathrm{Kcal}$, frugos 85 $\mathrm{Kcal}$, galletas $45 \mathrm{Kcal}$, gaseosas $120 \mathrm{Kcal}$ y algunas adolescentes consumen en su refrigerio algún tipo de fruta como mandarina $44 \mathrm{Kcal}$ ( 1 unidad), plátano $108 \mathrm{Kcal}$ (1 unidad) y naranja $38 \mathrm{Kcal}$ (1 unidad). Alimentos que no cubren el requerimiento nutricional necesario para un refrigerio y mucho menos en reemplazo del desayuno.

Así como el refrigerio forma parte de la alimentación de las adolescentes, también el almuerzo contiene los alimentos vitales, esenciales en esta edad.

“.. Llego a mi casa, me cambio, almuerzo generalmente sopas, segundo (arroz con algún producto animal) y refresco (limonada o cebada), muy poco como frutas y verduras, porque no me agradan..." (Margarita).

\section{“...Llegando a mi casa caliento mi almuerzo que consta de sopa, arroz, pollo o pescado..." (Gardenia).}

El almuerzo es una oportunidad de recibir un aporte nutricional considerable (30\%) y es por esta razón por la que resulta importante observar su dimensión saludable. El almuerzo es el tipo de comida más completa, ya que incluye en la mayoría de los casos todos los grupos de alimentos: fruta, carnes, cereales, verduras, leguminosas y grasas. Se recomienda para mantener un peso saludable una porción abundante de verdura y una porción moderada de cereal (arroz o pasta), que las carnes sean magras ( $\sin$ grasa visible), las leguminosas como frijol o lenteja son una opción en caso de tener buen apetito, pues son fuente de proteína vegetal y fibra. Sin embargo, la mayoría de adolescentes, consumen una alimentación poco balanceada, ya que tienen preferencias por ciertos grupos de alimentos, como los que aportan carbohidratos y grasas, y en menor proporción los que aportan proteínas, vitaminas y minerales, en particular las menestras y leguminosas, asi como las frutas y verduras.

En el estudio de caso se encontró, que las adolescentes además de no consumir una buen almuerzo, suprimen la merienda o lonche, ya sea por el desconocimiento de su importancia, por escasos recursos económicos y/o por influencias o costumbres de la familia; esto puede generar que el adolescente no reciba el aporte adecuado a sus requerimientos nutricionales ideales; pues según Mataix Verdu, la merienda debe aportar el $10 \%$ de los requerimientos nutricionales totales (2400 2600 Kcal):10 así mismo, Jury menciona "el principal aporte de la merienda es llegar con menos hambre a la comida programada y permitir al organismo generar un ritmo menos ansioso". Además, la merienda supone un paréntesis y una recarga de energía muy útil y necesaria para continuar la jornada o para emprender una serie de actividades que requieren un esfuerzo psíquico y/o físico.

Después de la merienda, la última comida del día es la cena, donde se considera consumir alimentos ligeros; al respecto, las adolescentes manifestaron lo siguiente:

“...ceno a las 8.00 generalmente lo mismo del almuerzo pero en poca cantidad... No realizo ningún tipo de dieta, pero a veces como: hamburguesas, salchipapa, porque por mi casa las preparan bien ricas o mi mamá me prepara diferentes frituras..." (Rosa). 
“...En la cena tomo una taza de café, dos panes con mantequilla...no consumo comida chatarra porque me hace daño por mi gastritis..." (Gardeña).

El consumo de la cena no debe dejar de estimarse, pues gracias a esta comida, durante el sueño se da el proceso de fijación de los minerales y nutrientes, que servirán para que las personas despierten con energías. Pero muchas veces la cena, no suele apreciarse como es debido, no sólo desde el punto de vista nutritivo, sino que también desde el punto de vista social. Las adolescentes son las que se ven más afectadas por este hecho. En primer lugar, a nivel de nutrición, la cena cada vez más se está convirtiendo en un desorden alimenticio, en el sentido de que se comen alimentos chatarra y cargados de grasas. Esto genera que los nutrientes que aporta los alimentos que se consumen en la cena, sean bastante negativos para el organismo.

Según Mataix Verdu, la cena debe aportar un 20\% del total de requerimientos nutricionales (2400 $2600 \mathrm{Kcal}),{ }^{10}$ es decir un aproximado de $500 \mathrm{Kcal}$; en este caso, las adolescentes consumen mayormente los mismos alimentos que del almuerzo o simplemente consumen hamburguesas, gaseosas o café con panes, los mismos que no son suficientes y en algunos casos exceden los requerimientos energéticos. Tal como se evidencia en la siguiente unidad de análisis:

\section{“...El año pasado estuve enferma y mi mamá me} llevó a Lima para que me vean porque estaba muy mal, ella me ayudaba con mi dieta que me dio el médico, me hizo bajar de peso por problemas del estómago, él médico me dijo que era porque comía comida chatarra; Antes si comía: hamburguesas, salchipapas casi todo los días, pero ahora ya no como mucho solo algunas veces..."(Violeta).

Una mala alimentación ocurre cuando tenemos un aporte insuficiente de uno o más nutrientes.

También podemos hablar de mala nutrición cuando hay un exceso de uno o varios nutrientes. Si una persona no satisface sus necesidades alimenticias diariamente se corre el riesgo de sufrir, problemas o trastornos nutricionales ${ }^{9}$.
Cualquier modificación en los hábitos y costumbres alimenticias se verá reflejada en la salud. Se debe consumir todo tipo de alimentos que nos aporten cantidades suficientes de hidratos de carbono, proteínas, grasas, vitaminas, minerales y agua. Estos son los nutrientes esenciales que se debe consumir para satisfacer la demanda de energía de nuestro cuerpo.

Así como la necesidad de alimentarse es fundamental para que una persona viva sanamente, también el cuerpo necesita descansar para reponer de la mejor manera a los gastos de energía diario, esto se discute en la siguiente sub categoría.

\subsection{Estilos de descanso y sueño.}

El sueño es un estado de reposo uniforme de un organismo. En contraposición con el estado de vigilia cuando el ser está despierto, el sueño se caracteriza por los bajos niveles de actividad fisiológica (presión sanguínea, respiración) y por una respuesta menor ante estímulos externos. EI sueño es alimento para el cerebro. Durante el sueño se desarrollan importantes funciones del organismo y de la actividad cerebral, y se recomienda que las adolescentes deben descansar un promedio de 6 a 8 horas $^{12}$.

Muchos jóvenes padecen trastornos de sueño que precisan tratamiento tales como; narcolepsia, insomnio, síndrome de piernas inquietas, apnea del sueño, etc. ${ }^{12}$ Respecto a este estilo de vida, las adolescentes manifestaron lo siguiente:

“...Me acuesto a la 1.00 ó 2.00 por ver televisión, una película interesante de 1 ó 2 horas o porque tengo tareas pendientes que me gusta que me queden bien, pero tardo en conciliar el sueño cuando estoy muy estresada sobre todo..." (Rosa).

“...Por lo general duermo desde las 10:00 pm, dependiendo si tengo muchas tareas o cosas que hacer y me despierto como a las 6:00 am, siempre trato de llevar esa rutina, los fines de semana 
duermo un poco más, ya que me levanto más tarde..." (Jazmín).

Como podemos evidenciar, las conductas de las adolescentes, con respecto al sueño es variada, algunas prefieren dormir sus 8 horas al día; en tanto que otras, no; incluso una mencionó, tener problemas para conciliar el sueño, cuando esta sobrecargada de tareas o preocupaciones.

No dormir lo suficiente o tener problemas con el sueño pueden tener serias consecuencias como: Limitar tu capacidad de aprendizaje, escucha, concentración y tu capacidad para resolver problemas. Puedes incluso olvidar información importante como nombres, números, tareas o una cita con alguien especial. La falta de sueño contribuye en la aparición de acné y otros problemas dermatológicos, también favorece conductas agresivas e inapropiadas como enfrentamientos con sus compañeros y tensiones con los profesores y familiares, conlleva a comer demasiado o comer de manera poco saludable, exceso de dulces y comida basura que contribuyen al aumento de peso.

Así también como el cuerpo y la mente necesitan descansar para reponerse de las actividades que realizan diariamente, también necesita de diversión y distracción para poder continuar con su desarrollo, esto se discute en la siguiente subcategoría.

\subsection{Estilos de divertirse}

La diversión es el uso del tiempo de una manera planeada para el refresco terapéutico del propio cuerpo o mente. Mientras que el ocio es más bien una forma de entretenimiento o descanso, la diversión implica participación activa pero de una manera refrescante y alegre ${ }^{13}$.

El umbral de cambio y de peligro gira en torno a los 13 años, para quienes son responsables de un adolescente. Esto se vincula al hecho de que a los 16 años es cuando, de acuerdo a los estudios realizados al respecto, se vuelven hábitos, las conductas que pueden perjudicar al joven y a su entorno. ${ }^{14}$

De acuerdo a las experiencias de las adolescentes que participaron en el estudio de caso, una forma de relajarse para ellas es saliendo a alguna fiesta con sus pares e ingerir bebidas alcohólicas incluso, tal como se evidencia en la siguiente unidad de análisis.

“...En un fin de semana no salgo porque ya no me gusta, antes si salía a fiestas o a discotecas, más o menos 2 veces por semana, iba con mis amigas, pero nunca a fumar, solo tomaba vino cuando se presentaba la ocasión, nunca salí a hacer otras cosas incorrectas como consumir drogas, como otras chicas por mi casa que sí lo hacen...ahora prefiero quedarme en mi casa a leer o estudiar y luego por las tarde voy a ayudarla a mi hermana a su trabajo...."(Violeta).

"...En la noche no salgo, solo a reuniones familiares, pero no fumo, brindo cuando es la ocasión y muy rara vez salgo con amigas de noche..." (Margarita).

"...Por las noches salgo a la discoteca con mis amigas..." (Jazmin)

Por lo general, en el estudio, casi todas las adolescentes manifestaron lo mismo, que se divierten con sus amigos y amigas en fiestas, por diversos motivos, a la par que beben licores en cantidades moderadas y muy pocas veces se divierten en reuniones familiares, con los suyos.

A la vez que el ser humano necesita divertirse, también es necesario realizar actividad física que ayuda a mantenerse saludables $y$ en buen funcionamiento físico y mental.

\section{4.- Estilos de realizar actividad física y deporte}

La actividad física es esencial para el crecimiento, el desarrollo y la salud de los jóvenes. Mantenerse en movimiento regularmente, mejora la capacidad cardiovascular, la resistencia, la flexibilidad y la 
densidad ósea, además de ayudar a mantener un peso saludable, la actividad física mejora la autoestima, el rendimiento escolar, el bienestar emocional y cognitivo.

Los pocos espacios en los hogares incluso en la propia comunidad de que disponen las adolescentes para realizar una adecuada actividad física, genera que lo practiquen solo en horas académicas, lo cual se verifica en las siguientes unidades de análisis:

“...solo realizamos deportes en el colegio en los cursos que nos toca y luego salimos al recreo a distraernos, caminamos por el patio o nos sentamos en el aula a conversar de diferentes temas, de los trabajos, algún programa o de algo que nos pasó..." (Violeta).

"...realmente solo hago educación física en el colegio, no dispongo de tiempo y no le doy la importancia que quizás la tiene..." (Rosa).

Las actividad y deporte en las adolescente varía según el lugar en donde se encuentran, por ejemplo en el colegio solo tienen a de 1 a 2 horas de educación física como parte de un curso, en el que solo las adolescentes se abocan a ello tal como lo evidenciamos en la unidad de análisis anterior y que a veces estas a pesar del poco tiempo no lo realizan por los diferentes motivos generalmente de salud o de espacio o por el clina en el que estamos.

En lo que respecta a actividad física y deporte fuera de la institución educativa, se evidencia que la mayoría de ella son sedentarias por diversos motivos tal y como se demuestra en las entrevistas.

La actividad física es fundamental para el crecimiento y desarrollo del ser humano, formando parte importante en su vida, pero a la vez la persona debe de organizar y disponer del tiempo libre que le queda después de realizar sus actividades principales, es por ello que se ha creado la siguiente sub categoría. 15

\section{5.- Estilos de disponer el tiempo libre}

Se denomina tiempo libre a aquel tiempo del que disponemos las personas fuera del dedicado a nuestras obligaciones, ya sean laborales, educativas, familiares, etc. Este tiempo del que podemos disponer libremente lo podemos malgastar o aprovechar de una forma creativa $y$ enriquecedora. La sociedad moderna ha establecido una relación entre ocio y consumo, evitando otras alternativas de ocio que favorezcan la creatividad y enriquezcan nuestra personalidad. El ocio nos ofrece alternativas enriquecedoras desde el punto de vista de la persona como un ser social, en este sentido se pueden englobar multitud de actividades que podemos realizar dentro de un grupo, desde talleres de teatro, actividades deportivas dentro de un equipo o un club, actividades musicales, perteneciendo a un grupo o una banda, etc. Otras actividades nos enriquecen como ser individual, como pueden ser la lectura, o la práctica de algún tipo de hobby como la pintura, etc. ${ }^{13}$

Las adolescentes entrevistadas, refirieron lo siguiente, con respecto al uso del tiempo libre:

"en mis tiempos libres, veo televisión, algunas novelas 1 o 2 horas y luego realizo tareas desde las $5 \mathrm{pm}$ hasta la noche; Me voy al internet a sacar mis trabajos y me demoro más o menos 2 horas..."(Violeta).

“...Después de almorzar, veo televisión 1 hora, realizo mis tareas, de 3 o 4 horas, utilizo el internet en casa para mis trabajos, 2 horas...mayormente nos encontramos en el colegio por la mañana para realizar las tareas o trabajos grupales en mi casa o en casa de alguna de ellas"...(Margarita).

"...Por la tarde lavo mi ropa, después veo la televisión dos horas...luego me pongo a estudiar, después voy al internet una hora a realizar algunos trabajos y a chatear..."

(Clavel) 
La recreación constituye un derecho fundamental del ser humano de acuerdo con la Organización de las Naciones Unidas, es la sexta necesidad básica después de la nutrición, la educación, la vivienda, el trabajo y la seguridad social, constituyendo un medio de unidad integral e integradora que promueve el desarrollo intelectual, emocional, físico y psicológico del individuo. Entendemos por tiempo libre el tiempo disponible, es decir, el que no utilizamos para trabajar, comer o dormir. ${ }^{15}$

El tiempo libre tiene una capacidad virtual, es tiempo a nuestra disposición que podemos utilizar adecuadamente o malgastar. El buen o mal uso del tiempo libre es una cuestión estrictamente personal, porque con el ritmo de trabajo de los padres de familia y la intensa vida diaria, pocos jóvenes se sienten seguidos por sus progenitores en su tiempo libre ${ }^{15}$.

\section{Conclusiones}

En el estudio realizado, las adolescentes aportan diferentes conceptualizaciones de estilos de vida, según sus conocimientos, juicios y prácticas cotidianas. En su totalidad las adolescentes relacionan a los estilos de vida con: una adecuada alimentación, descanso y sueño, realización de ejercicio físico y el buen uso del tiempo libre, lo que coincide con el concepto dado por la National Health Service, que señala que los estilos de vida, son el conjunto de actitudes, conocimientos $y$ comportamientos que determinan nuestra manera de vivir.

El estilo de alimentación, en el grupo de adolescentes que participaron del estudio, se caracteriza de manera general por el consumo de tres comidas: el desayuno, el almuerzo y la cena; sin embargo, la gran mayoría de ellos, omiten el desayuno por falta de tiempo, el refrigerio es a base de comida chatarra y la cena se caracteriza por que consumen la comida del almuerzo o simplemente lo reducen a un lonche (café con panes y algún acompañante), incluso muchas veces con el predominio de frituras. En contraposición a una alimentación equilibrada, que según este grupo etareo, consta de tres comidas: desayuno, almuerzo, cena, y dos refrigerios, entre el medio día y la media tarde.

Con respecto al estilo de vida, de descanso y sueño, las adolescentes presentan una diversidad de patrones, con la prevalencia de sueño y descanso limitado; ya que no son conscientes de su gran importancia en el mantenimiento de la salud. El sueño es un factor importante dentro de la vida, pues un buen descanso ayuda a un adecuado desarrollo de todo el cuerpo, en este caso se ve interrumpido por la rutina de las actividades diarias y por el desarrollo de las responsabilidades como estudiantes de secundaria, lo que genera un patrón de descanso y sueño inadecuados, con sus consecuentes problemas: cansancio, falta de concentración en clases, desgano, apatía y falta de ánimo para realizar actividades.

La adolescencia es una etapa en la que requieren mayor actividad física, pero a pesar de esto se evidencia que las adolescentes no la realizan adecuadamente, es decir ha sido reemplazado por el facilismo de la tecnología: videojuegos, internet, amigos, por la modernidad de usar escaleras eléctricas, ascensores, etc. lo que genera un mal estilo de vida, en la que prima el sedentarismo, de la mano con problemas de sobrepeso y obesidad, ante la falta actividad física. Para este grupo de estudio, la actividad física lo limita solo al cumplimiento de ejercicios y deporte, dentro de la asignatura que les brinda su plan estudios.

Las adolescentes, tienen la concepción de que el estilo de vida diversión, hace referencia a la combinación de momentos de relax con el consumo de bebidas alcohólicas, ya sea en reuniones con sus pares (salidas a discotecas y fiestas) o en reuniones familiares, excluyendo por completo cualquier otro tipo de actividad recreativa en este estilo de vida, esto puede de verse a la falta de conocimiento, a los deficientes 
recursos económicos, o a la falta de tiempo, para realizar di versas actividades recreativas.

Finalmente con respecto al uso del tiempo libre, en el grupo de estudio existe un predominio de utilizarlo en juegos de internet, en ver televisión y en la realización de tareas del colegio; ninguno mencionó el uso de su tiempo libre en actividades de mayor importancia como leer un periódico, una revista, salir con la familia, hacer deporte, cultivar el arte, etc. Esto hace pensar que los hábitos que vienen cultivando este grupo de adolescentes, no son adecuados, ni por tanto, saludables.

\section{Bibliografía}

1. Organización mundial de la salud .Estilos de vida saludable. Disponible en http://www.who.int/es/

2. Instituto Nacional de estadística e informática. Estado de la niñez en el Perú.

Lima - Febrero 2010. Disponible en: http:/www.minedu.gob.pe/digesutp/formacionini cial/wpdescargas/bdigital/037estadoniñezperu20 12.pdf

3. Jensen J. Características de la salud en la etapa del adolescente. EE.UU. 2010.

4. Polit D, Hungler B. Investigación científica en ciencias de la salud: Principios y métodos. Ed. 5ta; 2000.

5. Menga L, Marli E. El estudio del Caso: Su potencial en Educación. 1986.

6. Nut beam D. Aaro L y Carford J. Understanding children's health behavior: The impication for health promotion for young people. Social Science \& Medicine; 1989.

7. Ramos M. Tesis doctoral: Estilos de vida y salud en la adolescencia.2010. Disponible en:http://www.injuve.es/observatorio/tesis doctorales/estilos-de-vida-y-salud-en-la adolescencia

8. Medellín G. Crecimiento y desarrollo del ser humano, Tomo I. Edit. : Guadalupe.

9. Campos P. Sedentarismo en los adolescentes: Enemigo silencioso; 2011. Disponible en: http://www.guioteca.com

10. Mataix J. Nutrición y alimentación humana. Edición: $1^{\text {a }}$ Lengua: castellano Año; 2002.

11. Ángeles A, Pinto J. Instituto de salud pública. Nutrición y salud: El desayuno saludable. Madrid.

12. Cinteco. Psicología clínica y psiquiátrica. Madrid; 2005-2012.

\section{Aparicio T. Puleva salud. Psicóloga.} Especialista en infancia y adolescencia. Granada; 2010.

14. Gómez C. A partir de los 13 años, los hábitos negativos pueden fijarse en los adolescentes. StarMedia. Disponible en: http://www.mujer.starmedia.com

15. Guerrero G. La recreación alternativa del desarrollo comunitario. Universidad Pedagógica Experimental Libertador. Venezuela; 2006. 